\title{
Optische Gassensorik mit chiral-nematischen Flüssigkristallen und koaxialem Elektrospinnen
}

\author{
Lukas Pschyklenk ${ }^{1}$, Thorsten Wagner ${ }^{2}$ und Peter Kaul ${ }^{1}$ \\ ${ }^{1}$ Institut für Sicherheitsforschung, Institut für Detektionstechnologien, Hochschule Bonn-Rhein-Sieg, \\ Rheinbach, Deutschland \\ ${ }^{2}$ Department Chemie, Universität Paderborn, Paderborn, Deutschland \\ Kontakt: lukas.pschyklenk@h-brs.de
}

\begin{abstract}
Zusammenfassung: Durch Dotierung eines nematischen Flüssigkristalles mit einer chiralen Substanz wird eine helikal strukturierte Phase induziert, die in der Lage ist, einfallendes Licht wellenlängenselektiv zu reflektieren. Bei der Reaktion des Dotiermittels mit einem gasförmigen Analyten verändern sich die Ganghöhe dieser Struktur und damit die reflektierte Wellenlänge. Liegt diese im Bereich des sichtbaren Lichts, ist eine Farbänderung mit dem menschlichen Auge zu beobachten. Es ist dabei sinnvoll den Flüssigkristall z.B. in einem Polymer einzukapseln, um ihn vor mechanischen Einflüssen und Umwelteinflüssen zu schützen. Eine Möglichkeit zur Einkapselung ist das koaxiale Elektrospinnen. Vorteile sind unter anderem die Realisierung einer großen Oberfläche und einer sehr geringen Wanddicke der schützenden Schale, die die Diffusion von Gasen durch die Wand hindurch ermöglicht. Um die Funktionsfähigkeit eines solchen Sensors zu testen, wurde ein $\mathrm{CO}_{2}$-sensitiver Flüssigkristall verwendet. Dieser wurde in eine Schale aus Polyvinylpyrrolidon (PVP) versponnen und die Reaktion mit $\mathrm{CO}_{2}$ spektroskopisch analysiert.
\end{abstract}

Schlagwörter: Optische Gassensorik, Chiral-nematischer Flüssigkristall, Koaxiales Elektrospinnen

\section{Einleitung}

Sensoren nehmen einen immer größer werdenden Stellenwert in unserem Leben ein. Ob im Auto, im Haushalt oder in der Industrie, überall befinden sich Sensoren, die uns schützen, die Effizienz von Maschinen verbessern oder uns die Interaktion mit diversen Geräten ermöglichen. Besonders interessant ist der mobile Einsatz von Gassensoren, da dort oft eine Batterie die zur Verfügung stehende Energiemenge begrenzt. Gassensoren, die keinen oder nur einen sehr geringen Stromverbrauch aufweisen, könnten neben einer klassischen Anwendung im Brandschutz, z.B. auch in der Textilindustrie zum Einsatz kommen. In Textilien eingearbeitete Sensoren könnten als Teil der persönlichen Schutzausrüstung durch einen Farbumschlag das Vorhandensein eines Gases oder eine Überschreitung des Grenzwertes toxischer Substanzen anzeigen. Eine Möglichkeit diese Kriterien zu erfüllen sind optische Gassensoren, die eine selektive Reflektion aufweisen, wie in Abbildung 1 dargestellt.

Anders als bei den gängigen resistiven Gassensoren [1] funktioniert das hier vorgestellte Sen- sorprinzip bei Raumtemperatur und kommt grundsätzlich ohne Stromversorgung aus.

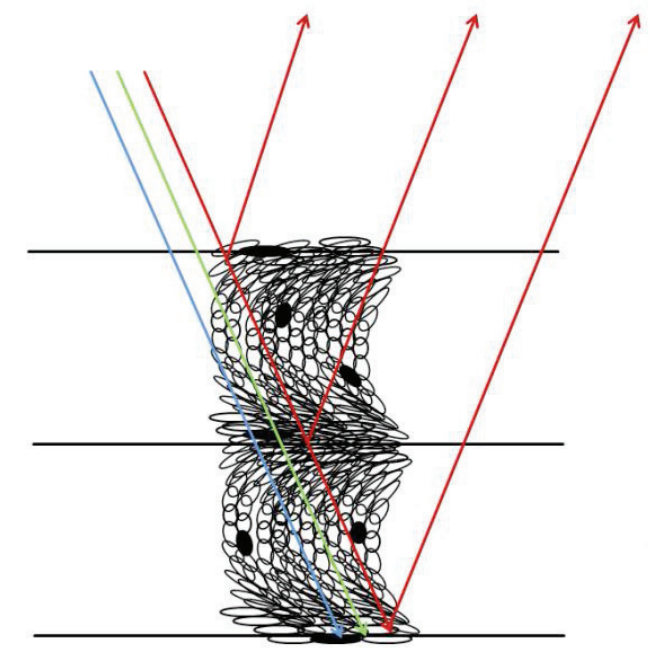

Abbildung 1: Prinzip der wellenlängenselektiven Reflektion an der chiral-nematischen Flüssigkristallstruktur 


\section{Das Sensorprinzip}

Um eine selektive Reflektion zur erreichen, wird ein nematischer Flüssigkristall mit einer chiralen Substanz dotiert. Das Dotiermittel induziert eine helikale Struktur [2], deren Ganghöhe $\boldsymbol{p}$ (engl. Pitch) von dem Verdrillungsvermögen (engl. helical twisting power, HTP) des Dotiermittels abhängt (Abbildung 2).

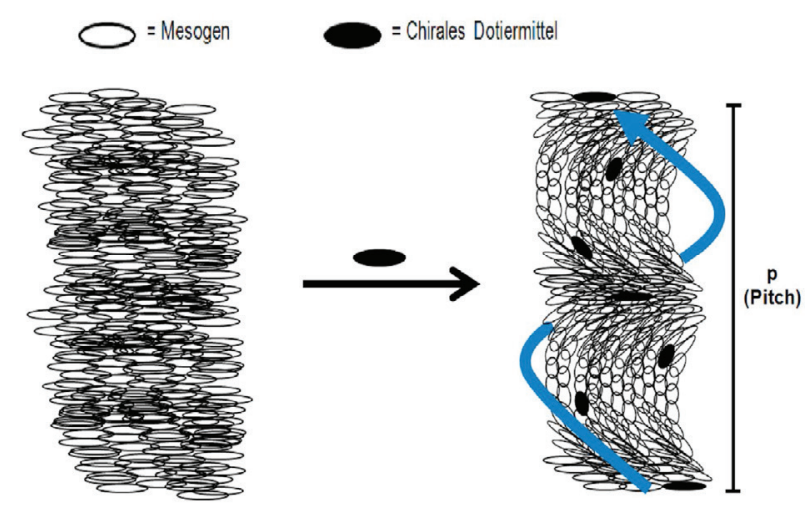

Abbildung 2: Induktion einer chiral-nematischen Phase durch Dotierung mit einer chiralen Substanz

Durch eine chemische Reaktion des Analyten mit dem Dotiermittel werden die HTP und somit die Ganghöhe verändert. (Abbildung 3)

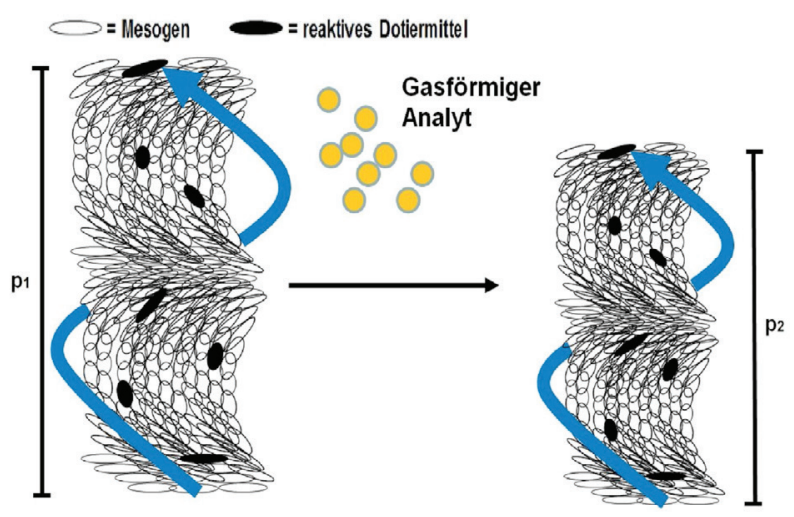

Abbildung 3: Reaktion des Dotiermittels mit einem Analyten und Änderung der Ganghöhe $p$

Han et al. [3] stellten einen $\mathrm{CO}_{2}$-sensitiven Sensor her, indem sie ein nematisches Flüssigkristallgemisch mit einem enantiomerenreinen Komplex aus einem TADDOL-Derivat und einem Diamin dotierten. Die induzierte helikale Überstruktur im Flüssigkristall, die sogenannte chiral-nematische Phase, hat außergewöhnliche optische Eigenschaften. Üblicherweise erscheint ein Gegenstand in einer bestimmten Farbe, weil ein oder mehrere Wellenlängenbereiche absorbiert werden und das menschliche Auge das Restspektrum empfängt.
Chiral-nematische Flüssigkristalle erscheinen hingegen in einer bestimmten Farbe, weil nur ein Wellenlängenbereich reflektiert wird. Das reflektierte Wellenlängenmaximum dieses Bereiches ist abhängig von der Ganghöhe $\boldsymbol{p}$ der Helix, dem Einfallswinkel $\boldsymbol{\theta}$ und dem mittleren Brechungsindex $\overline{\boldsymbol{n}}$ und kann mit $\boldsymbol{\lambda}=\boldsymbol{p} * \overline{\boldsymbol{n}} * \cos \boldsymbol{\Theta}$ [4] beschrieben werden.

Die Reaktion des Analyten mit dem Dotiermittel führt zu einer Änderung der Ganghöhe und somit zu einer sichtbaren und messbaren Verschiebung der reflektierten sowie der transmittierten Wellenlänge. Neben dem reversiblen System für $\mathrm{CO}_{2}$ stellten Han et al. auch ein irreversibles System für die Detektion von $\mathrm{O}_{2}$ vor [3], welches auf der Oxidation eines als Dotiermittel eingesetzten Dithiols zum Sulfid basiert. Ein weiterer $\mathrm{H}_{2} \mathrm{O}$-sensitiver Sensor wurde von Saha et al. entwickelt. [5]. Das dabei eingesetzte Dotiermittel, ein Alkoxysilan, wird direkt durch den Analyten in $\mathrm{SiO}_{2}$ und einen Alkohol gespalten. Daneben gibt es noch eine Reihe weiterer Publikationen [6-9], bei denen die geordneten Mesogene über eine Acrylatgruppe polymerisiert wurden. Die Sensorreaktionen basieren meist auf einem Quellvorgang durch Gasmoleküle, die in das Polymer diffundieren, und der damit verbundenen Vergrößerung der Ganghöhe, oder auf Nebenvalenzbindungen.

Die Anwendung des Sensorprinzips mit niedermolekularen Flüssigkristallen scheiterte bisher an der Beschaffenheit des Flüssigkristalles an sich. Es handelt sich um ein Fluid, welches flächig appliziert auf einem Substrat keinerlei mechanischen Schutz bzw. Schutz vor Umwelteinflüssen und Verschmutzung aufweist. Die Einkapselung dieser Flüssigkristalle scheint hier die geeignete Lösung zu sein. Wird der Flüssigkristall zum Schutz mit einem Polymerfilm abgedeckt, muss das zu analysierende Gas zunächst durch die Polymerschicht hindurchdiffundieren. Eine Schicht zum Zwecke des mechanischen Schutzes wird aber eine Dicke haben, die einer schnellen Sensorreaktion entgegensteht. Kim et al. schlugen eine Einkapselung des flüssigkristallinen Sensorsystems mittels koaxialem Elektrospinnen vor [10]. Lagerwall, Scalia und Enz [11-15] konnten bereits die koaxiale Verspinnbarkeit niedermolekularer Flüssigkristalle in PVP aufzeigen und deren Eigenschaften innerhalb der Fasern beschreiben. 
Das Elektrospinnen ist eine Methode zur Herstellung von Fasern mit Durchmessern im Mikro- bis Nanometerbereich. Dabei wird typischerweise eine Polymerlösung über eine Spritzenpumpe durch eine elektrisch leitende Kanüle gepumpt, an der eine Hochspannung angelegt ist. Durch die Hochspannung erfolgt innerhalb der Lösung eine Ladungstrennung, wobei sich positive Ladungen an der Spitze des an der Kanüle hängenden Tropfens sammeln. Bei Überschreitung einer kritischen Spannung formt sich ein Jet, der in Richtung einer wenige Zentimeter entfernten Gegenelektrode beschleunigt wird. Während dieser Flugphase unterliegt der Jet einigen Instabilitäten, die durch inhomogene Ladungsverteilung an der Oberfläche hervorgerufen werden. Diese Instabilitäten sorgen für eine Biegung und Streckung und somit für eine Abnahme des Jetdurchmessers sowie eine schnelle Verdunstung des Lösemittels durch die gleichzeitige Vergrößerung der Oberfläche. Sind die Prozessparameter richtig abgestimmt, sammeln sich auf der Oberfläche der Gegenelektrode feste Polymerfasern [16].

Das koaxiale Elektrospinnen unterscheidet sich von diesem Verfahren dahingehend, dass man zwei konzentrisch ineinander gesteckte Kanülen verwendet, die von zwei verschiedenen Fluiden durchströmt werden (Abbildung 4).

Man erhält Fasern, die aus einem Schalen- und einem Kernmaterial bestehen. Durch Verwendung eines amorphen Polymers als Schalen- und dem dotierten Flüssigkristall als Kernmaterial, lässt sich die Sensorreaktion durch die Polymerschale hindurch beobachten.

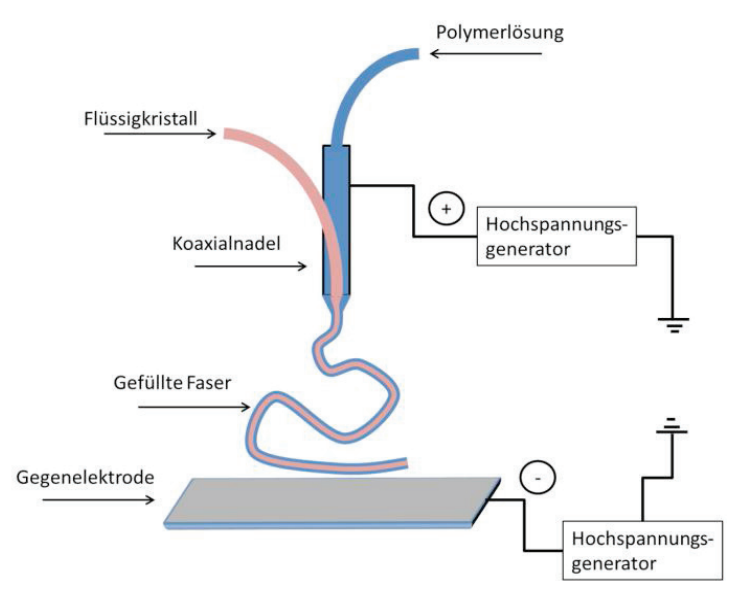

Abbildung 4: Schematische Darstellung des koaxialen Elektrospinnens mit flüssigkristallinem Kern

\section{Methoden und Materialien}

Das Flüssigkristallgemisch E7 (Synthon Chemicals) wurde mit einem 1:1 Gemisch aus dem TADDOL-Derivat $\quad\left((4 R, 5 R)-2,2-D i m e t h y l-\alpha, \alpha, \alpha^{\prime}, \alpha^{\prime}\right.$ tetraphenyldioxolan-4,5-dimethanol,Sigma Aldrich) und dem Diamin ((1R,2R)-(+)-1,2-Diphenylethylendiamin, Sigma Aldrich) durch direkte Zugabe der beiden Substanzen in einer Konzentration von 1,6Mol- $\%$ dotiert. Die Ganghöhe wurde mit der Grandjean-Cano-Methode $[17,18]$ bestimmt. Dabei wurden eine plankonvexe Linse und ein Objektträger verwendet, die beide mit einer Polyvinylalkoholschicht beschichtet und uniaxial orientiert wurden. Die Disklinationslinien der chiral-nematischen Phase wurden mit einem Auflichtmikroskop (Keyence VHX-600, Objektive: VH-Z100UR, VHZ500R) ausgemessen.

Für das koaxiale Elektrospinnen wurde eine Lösung aus 20 Gew.-\% PVP in Rotisol ${ }^{8}$ (Carl Roth) angesetzt. Durch diese hohe Konzentration konnten Fasern mit Durchmessern von über $5 \mu \mathrm{m}$ hergestellt werden, was die Auswertung mit einem optischen Mikroskop erleichterte. Durch Zugabe von $0,1 \mathrm{Gew} .-\% \mathrm{NaCl}$ wurde zudem die häufig beim Elektrospinnen auftretende Perlenbildung minimiert. Die Spannung betrug $15 \mathrm{kV}$, der Elektrodenabstand $20 \mathrm{~cm}$. Als Gegenelektrode diente ein Ring aus Aluminium.

Für die Auswertung der Sensorreaktion wurde eine Messkammer verwendet, mit der sowohl der besponnene Ring als auch der mit Flüssigkristall beschichtete Objektträger in Transmission unter Gaseinfluss spektroskopisch analysiert werden konnten (Abbildung 5).

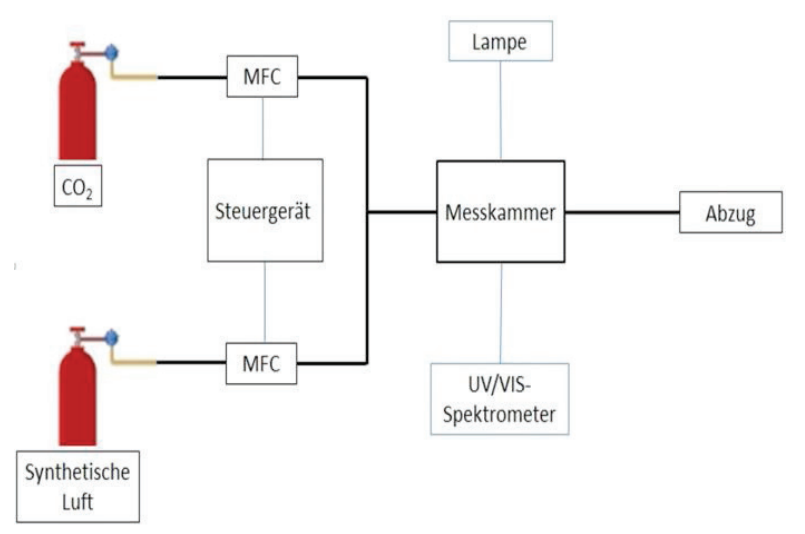

Abbildung 5: Aufbau zur spektroskopischen Messung der Sensorreaktion 
Über die beiden Massflowcontroller (MFC) ist eine $\mathrm{CO}_{2}$-Konzentration zwischen $2 \%$ und $100 \%$ in synthetischer Luft einstellbar. Während der ersten Versuche wurde mit einer $100 \%$ igen $\mathrm{CO}_{2}-$ Konzentration gearbeitet, da hier nur die grundsätzliche Funktionsfähigkeit der eingekapselten Sensoren untersucht wurde.

Als Lichtquelle diente eine Quarz-Halogenlampe (Fa. Dolan-Jenner, Typ MI150). Zur spektroskopischen Messung wurde mit einem USB2000Spektrometer der Fa. Ocean Optics bei einer Integrationszeit von $3 \mathrm{~ms}$ gearbeitet. Mit dem Starten des $\mathrm{CO}_{2}$-Flusses $(200 \mathrm{~mL} / \mathrm{min})$ wurden in fünfSekunden Abständen Spektren aufgezeichnet.

\section{Ergebnisse}

Die Auswertung mittels Grandjean-Cano-Methode ergab für die Dotierung mit 1,6Mol-\% eine Ganghöhe $p$ von $360 \mathrm{~nm}$. Bei senkrechtem Lichteinfall $\left(\Theta=0^{\circ}\right)$ und einem geschätzten mittleren Brechungsindex $\bar{n}$ von 1,6 [19] befindet sich das errechnete reflektierte Wellenlängenmaximum $\lambda$ bei $576 \mathrm{~nm}$ und entspricht auch in etwa den spektroskopisch gemessenen Werten, da in diesem Bereich ein Transmissionsminimum gemessen wurde. Die Messungen des reaktiven Flüssigkristalls auf dem Objektträger zeigten in Anwesenheit von $\mathrm{CO}_{2}$ eine deutliche Verschiebung des Transmissionsminimums zu kleineren Wellenlängen hin (Abbildung 6).

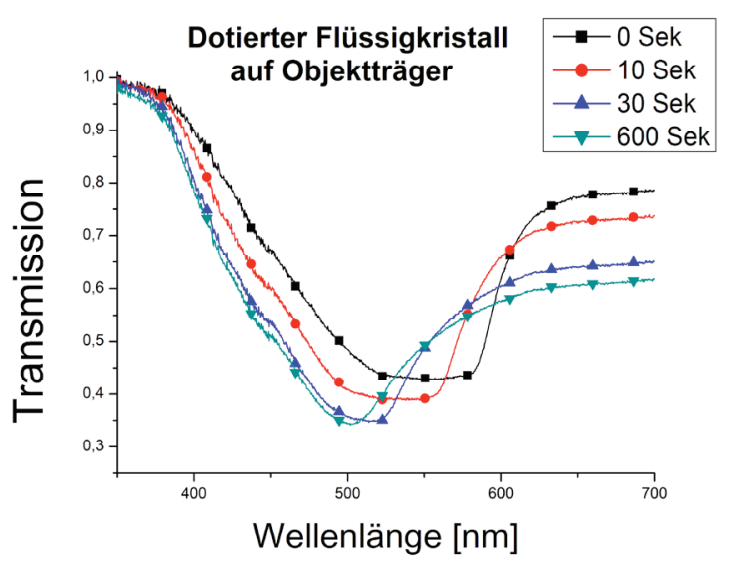

Abbildung 6: Verschiebung des Transmissionsminimums infolge der Reaktion mit $\mathrm{CO}_{2}$ auf dem Objektträger

Die koaxial gesponnenen Fasern haben einen Durchmesser zwischen $5 \mu \mathrm{m}$ und $7 \mu \mathrm{m}$, wobei der flüssigkristalline Kern einen Durchmesser von 2-3 $\mu \mathrm{m}$ aufweist. (Abbildung 7)

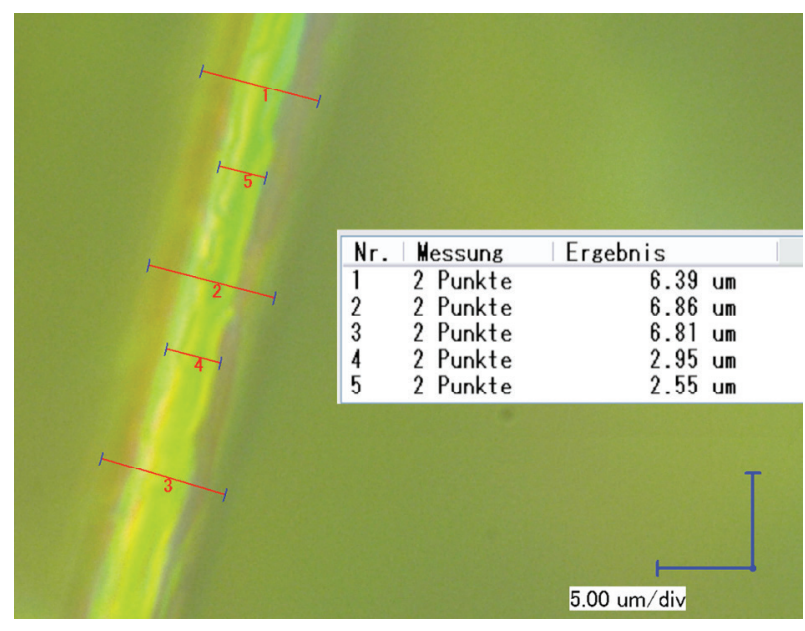

Abbildung 7: Lichtmikroskopische Aufnahme einer PVPFaser mit dotiertem, flüssigkristallinem Kern

Zur Untersuchung der Reaktion innerhalb der gefüllten Fasern wird das Spektrum vor dem Kontakt mit $\mathrm{CO}_{2}$ als Referenz genommen und die weiteren Spektren von diesem subtrahiert. Abbildung 8 zeigt die Änderung des Spektrums im Verlauf der Reaktion. Die gegenläufige Änderung der Intensitäten im Bereich von $500 \mathrm{~nm}$ bis $600 \mathrm{~nm}$ deutet auch hier auf eine Blauverschiebung des Transmissionsminimums hin.

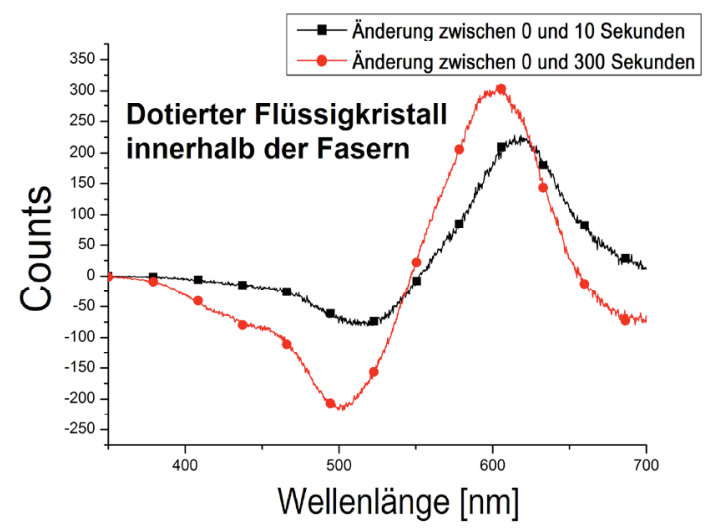

Abbildung 8: Änderung der Intensität infolge der Reaktion des Dotiermittels mit $\mathrm{CO}_{2}$ innerhalb der Fasern

Eine Änderung der Reflexions- und Transmissionseigenschaften lässt sich mit dem bloßen Auge feststellen. Hierzu wurde ein Vlies aus gefüllten PVP-Fasern halbiert und nur eine der Hälften mit $\mathrm{CO}_{2}$ in Kontakt gebracht. Abbildung 9 zeigt das Ergebnis dieses Experiments. 


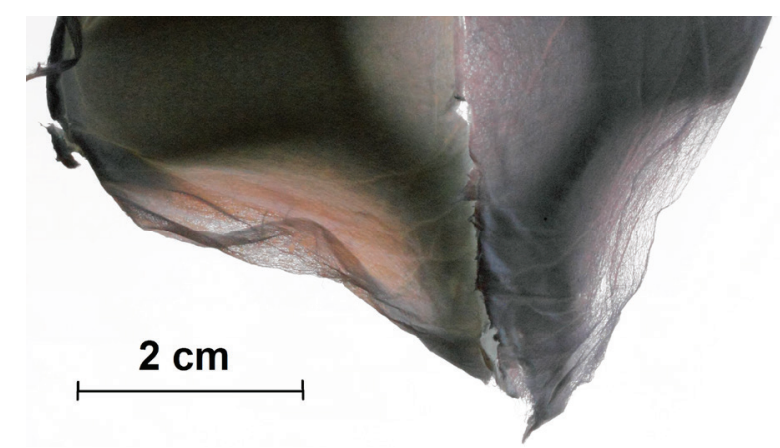

Abbildung 9: Fotographie zweier Vlieshälften in Transmission (Sonnenlicht). Links: Nach Kontakt mit $\mathrm{CO}_{2}$, rechts: Ohne Kontakt mit $\mathrm{CO}_{2}$

Die linke, mit $\mathrm{CO}_{2}$ in Kontakt gebrachte Hälfte zeigt eine Orangefärbung des Vlieses, während die rechte, unbehandelte Hälfte dem Beobachter in einer violetten Farbe erscheint.

\section{Diskussion}

Der von Han et al. beschriebene $\mathrm{CO}_{2}$-sensitive Flüssigkristall hatte sein Transmissionsminium vor dem Kontakt mit $\mathrm{CO}_{2}$ bei einer Wellenlänge von $637 \mathrm{~nm}$ und lag nach der Reaktion bei $495 \mathrm{~nm}$ [3]. Eine so deutliche Reaktion wurde hier nicht erreicht, insbesondere nicht bei den spektroskopischen Untersuchungen der Farbänderung innerhalb der Fasern. Die Änderung des Spektrums zwischen $500 \mathrm{~nm}$ und $600 \mathrm{~nm}$ im Verlauf der Reaktion belegen aber die generelle Funktionsfähigkeit solcher Sensoren. In weiteren Versuchen muss die Reaktionsgeschwindigkeit des koaxial gesponnenen Sensors mit der des offen applizierten verglichen werden. Es konnte aber bereits jetzt gezeigt werden, dass sich diese nicht gravierend unterscheiden und eine erste Reaktion schon nach wenigen Sekunden sichtbar ist. Wegen der variierenden Dicke des Vlieses ist es sinnvoll, bei künftigen Untersuchungen das Reflexionsspektrum statt des Transmissionsspektrums zu messen. Die fotographische Aufnahme des Vlieses deckt sich mit den spektroskopischen Beobachtungen. Das Vlies zeigt im Bereich von etwa $500 \mathrm{~nm}$ (grün-blau) nach der Reaktion mit $\mathrm{CO}_{2}$ ein Transmissionsminimum und somit ein Reflexionsmaximum im selben Bereich. In Abb. 9 ist die Komplementärfarbe (orange) zu sehen.

Um die Praxistauglichkeit dieser Sensoren zu verbessern, ist der Ersatz des wasserlöslichen PVP durch andere transparente Polymere wünschenswert. Weitere offene Fragenstellungen sind der Einfluss der Porosität, der Temperatur und der
Einfluss des weichmachenden Effektes des Flüssigkristalles auf die Polymerfasern.

\section{Literatur}

[1] P. GRÜNDLER, Chemische Sensoren: Eine Einführung für Naturwissenschaftler und Ingenieure ; mit 27 Tabellen, Springer, Berlin [u.a.] 2004.

[2] F. VÖGTLE, Supramolekulare Chemie: Eine Einführung, 2nd ed., Teubner-Studienbücher Chemie, Teubner, Stuttgart 1992.

[3] Y. HAN et al., J. Am. Chem. Soc. 2010, 132 (9), 2961.

[4] H. STEGEMEYER, Liquid crystals, Topics in physical chemistry, vol. 3, Steinkopff; Springer, Darmstadt, New York 1994.

[5] A. SAHA et al., Chem. Commun. 2012, 48 (38), 4579.

[6] P. V. SHIBAEV, K. SCHAUMBURG, V. PLAKSIN, Chem. Mater. 2002, 14 (3), 959.

[7] C.-K. CHANG, BASTIAANSEN, CEES W. M., D. J. BROER, H.-L. KUO, Macromolecules 2012, 45 (11), 4550.

[8] N. HERZER et al., J. Am. Chem. Soc. 2012, 134 (18), 7608.

[9] J. E. STUMPEL et al., Advanced Optical Materials 2014, 2 (5), 459.

[10] D. K. KIM, M. HWANG, LAGERWALL, JAN P. F., J. Polym. Sci. Part B: Polym. Phys. 2013, 51 (11), 855.

[11] LAGERWALL, JAN P. F. et al., Chem. Commun. 2008 (42), 5420.

[12] E. ENZ, U. BAUMEISTER, LAGERWALL, JAN P F, Beilstein J. Org. Chem. 2009, 5.

[13] E. ENZ, J. LAGERWALL, J. Mater. Chem. 2010, 20 (33), 6866.

[14] G. SCALIA et al., Macromol. Mater. Eng. 2013, 298 (5), 583.

[15] E. ENZ, V. LA FERRARA, G. SCALIA, ACS Nano 2013, 7 (8), 6627.

[16] J. H. WENDORFF, A. GREINER, S. AGARWAL, Electrospinning: Materials, processing, and applications, Wiley- $\mathrm{VCH}$, Weinheim 2012.

[17] L. BERGMANN, K. KLEINERMANNS, C. SCHÄFER, T. DORFMÜLLER, Lehrbuch der Experimentalphysik: Zum Gebrauch bei akademischen Vorlesungen und zum Selbststudium, 2nd ed., Lehrbuch der Experimentalphysik, / Bergmann; Schaefer ; Bd. 5, Gruyter, Berlin [u.a.] 2006.

[18] R. CANO, Bull. Soc. Fr. Mineral. 1968, 91 (20)

[19] J. Ll et al., J. Display Technol. 2005, 1 (1), 51. 